\title{
The tissue distribution, metabolism and hepatotoxicity of benzoylurea pesticides in male Eremias argus after a single oral administration
}

\author{
Jing Chang a, b, Wei Li a , Peng Xu a, Baoyuan Guo a, Yinghuan Wang a , Jianzhong Li a , \\ Huili Wang a, * \\ a Research Center for Eco-Environmental Sciences, Chinese Academy of Sciences, Shuangqing RD 18, Beijing 100085, China \\ ${ }^{\mathrm{b}}$ University of Chinese Academy of Sciences, Yuquan RD 19 a, Beijing 100049, China
}

\section{H I G H L I G H T S}

- Diflubenzuron preferred to accumulate in the fat and brain.

- The flufenoxuron levels in all tissues were greater than $1.0 \mathrm{mg} \mathrm{kg}^{-1}$.

- The excretion of flufenoxuron in the faeces was 1.5 fold higher than diflubenzuron.

- The Cyp1a and Ahr genes can serve as biomarkers to assess the liver toxicity.

\section{A R T I C L E I N F O}

\section{Article history:}

Received 30 January 2017

Received in revised form 29 April 2017

Accepted 1 May 2017

Available online 3 May 2017

Handling Editor: David Volz

\section{Keywords:}

Diflubenzuron

Flufenoxuron

Metabolism

Bioaccumulation

Hepatotoxicity
G R A P H I C A L A B S T R A C T

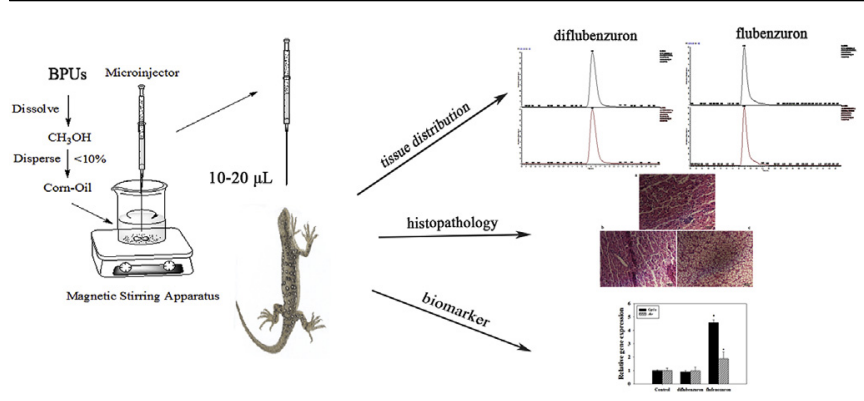

\begin{abstract}
A B S T R A C T
Benzoylurea pesticides (BPUs) are widely used to control the locust, but the toxicokinetics and hepatotoxicity of BPUs in lizards have not been investigated. In this study, the tissue distribution, metabolism and liver toxicity of diflubenzuron and flufenoxuron were assessed in the Eremias argus following a single oral exposure. Diflubenzuron preferred to accumulate in the fat and brain $\left(>1.0 \mathrm{mg} \mathrm{kg}^{-1}\right)$ and was rapidly eliminate in other tissues. In the liver, 4-chloroaniline was one of diflubenzuron metabolites, although with a concentration less than $0.05 \%$ of the accumulated diflubenzuron. No significant difference was observed in the liver histopathology between the control and diflubenzuron exposure group. The expressions of Cyp1a and Ahr gene which control the cell apoptosis were also equal to the control level. After flufenoxuron exposure, biomodal phenomenon was observed in the liver, skin, brain, gonad, kidney, heart and blood circulation was an important route for the flufenoxuron penetration. The concentrations of flufenoxuron in all tissues were greater than $1.0 \mathrm{mg} \mathrm{kg}^{-1}$ at $168 \mathrm{~h}$. The excretion of flufenoxuron in the faeces was 1.5 fold higher than diflubenzuron. The hepatocytes in the flufenoxuron treated group showed vacuolation of cytoplasm and decreased nucleus. In addition, the Cyp $1 a$ and Ahr genes were significantly up-regulated in the flufenoxuron exposure group. These results suggested that the higher hepatotoxicity of flufenoxuron may be attributed to the higher residual level in the lizard tissues and the Cyp1a and Ahr genes can serve as biomarkers to assess the liver toxicity.
\end{abstract}

๑) 2017 Elsevier Ltd. All rights reserved.

\footnotetext{
* Corresponding author.

E-mail address: huiliwang@rcees.ac.cn (H. Wang).
}

\section{Introduction}

The lizards are the largest family of reptiles, accounted for $71 \%$ of the reptile species (Gibbons et al., 2000). It has been regarded as an 
ideal model to assess the toxic effect of contaminants on the reptiles (Talent et al., 2002). The Mongolian racerunner (Eremias argus) is a small lizard species belonging to family Lacertidae, Reptilia. Eremias argus primarily inhabits grasslands near mountains, sand dunes formed along coastlines in the Korean peninsula, Mongolia, and certain areas of Russia and China (Kim et al., 2010).

In the grasslands, benzoylurea pesticides (BPUs) are widely used to control locust through inhibiting the chitin synthesis (Coppen and Jepson, 1996). Diflubenzuron is one of the early used BPUs, which could persistent in the grassland for two months (Symmons, 1992). The aerial application of diflubenzuron resulted in deposition levels ranging from 867.5 to $1824.4 \mathrm{ng} \mathrm{g}^{-1}$ (Rodriguez et al., 2001). Diflubenzuron has limited impact on the non-target invertebrate populations (Soltanimazouni, 1994) and low toxicity to birds (Whitmore et al., 1993).

Several other BPUs, such as flufenoxuron and teflubenzuron are now available as candidates in locust control for their enhanced toxicity compared with diflubenzuron (Clarke and Jewess, 1990). After leaching for $70 \mathrm{~d}$ in the soil, more than $99 \%$ of diflubenzuron was eliminated while flufenoxuron and novaluron remained at $>30 \%$ and 50\%, respectively (Hsiao et al., 2013). Lizards can contact with the pesticides through both oral and dermal routes. However, the toxic effect of BPUs to lizards has not been investigated.

The degradation of BPUs is mainly through cleavage of the urea bridge. 4-chloroanilian and 2,6-difluorobenzamide are two of the possible metabolites of BPUs (Fig. S1), which have been detected in the water (Rodriguez et al., 1998). 2,6-difluorobenzamide is detected on the foliage due to the photodegradation of diflubenzuron (Rodriguez et al., 2001). 4-chloroanilian is not the major metabolite in the fish and rat, but it is considered to be mutagenic, and possibly a human carcinogen (Olsvik et al., 2013).

The liver is one of the most sensitive organs to show alteration in biochemistry, physiological and structure following exposure to various types of environmental pollutants (Giari et al., 2007). The enzymes, especially cytochrome P450 (CYP) in the liver play an important role for the detoxification of contaminants. However, there is paucity of information regarding the impact of BPUs on the metabolic enzymes.

The aim of this study is to investigate the bioaccumulation, metabolism and hepatotoxicity of BPUs (diflubenzuron and flufenoxuron) in the Chinese native lizard (Eremias argus) after a single dose treatment. Uptake kinetics in the lizard tissues was examined. The hepatic index, liver histopathology and metabolism-related genes were measured to explore the toxic effect of BPUs in the lizard liver.

\section{Methods and materials}

\subsection{Chemicals}

Diflubenzuron, 4-chloroaniline and flufenoxuron (Fig. S1) were (purity 98\%) purchased from J\&K Scientific Ltd. (Beijing, China). The solvents including methanol, acetonitrile, and $n$-hexane (HPLC grade) were obtained from Dikma (Beijing, China).

\subsection{Animal husbandry}

We collected juvenile Eremias argus from the wild in the Inner Mongolia Province. We have maintained them in our laboratory for more than 4 years and established the reproduction method of Eremias argus (Wang et al., 2014). The 2-3-year old mature Eremias argus (3.5-4 g) were obtained from our breeding colony in Changping district, Beijing, China on 20th May. Due to the potential variability in dosage associated with vitellogenesis and egg production, females were not included in the exposure experiment.
The lizards were kept in $5.0 \times 1.2 \times 0.4 \mathrm{~m}$ solid bottom indoor aquariums. Ultraviolet lamps were set on $12 \mathrm{~h}: 12 \mathrm{~h}$ light/dark cycles to provide enough light and maintain the needed temperature. The temperature and humidity were maintained at $25-30{ }^{\circ} \mathrm{C}$ and $30-50 \%$, respectively. The lizards were fed with live mealworms (Tenebrio molitor) twice a day and the lizard excreta were removed every other day.

\subsection{Exposure experiment and sample collection}

The data regarding the exposure and toxic effects of BPUs on lizards are lack. Birds have been used as a surrogate for reptiles to assess the risk of pollutants (Weir et al., 2010). The $\mathrm{LD}_{50}$ of diflubenzuron and flufenoxuron is greater than $2000 \mathrm{mg} \mathrm{kg}^{-1}$. In this study, $1 \%$ of $\mathrm{LD}_{50}$ value $\left(20 \mathrm{mg} \mathrm{kg}^{-1}\right.$ ) was selected as the exposure concentration for lizards.

Before experiment, the lizards were allowed to acclimate in the experimental glass cages $(60 \times 60 \times 40 \mathrm{~cm})$ for one week, and then separated randomly into the control and exposure groups (total 3 groups, control group $n=6$, exposure groups $n=48$ ). The lizards in the exposure groups were orally administered $(10-20 \mu \mathrm{L})$ with $20 \mathrm{mg} \mathrm{kg} \mathrm{kg}^{-1}$ diflubenzuron or flufenoxuron (diluted by corn oil) once. In the control group, only methanol diluted with corn oil was dosed. In the exposure group, the lizards were killed at 1, 3, 6, 12, $24,72,120,168 \mathrm{~h}$ after dosing. In the control group, the lizards were only sacrificed at $168 \mathrm{~h}$ because of its non distinctiveness among different time points (Chang et al., 2016). The body weights of lizards were measured. The blood was immediately centrifuged at $2500 \times \mathrm{g}$ for $10 \mathrm{~min}$, and upper phase (plasma) was collected. The liver, brain, kidney, heart, skin, fat, and gonad were weighted and then frozen at $-20{ }^{\circ} \mathrm{C}$. A part of liver was stored in $4 \%$ paraformaldehyde or RNA store. Two lizards were selected randomly from each group, and three replicates were prepared. The faeces of the lizards were also collected during $7 \mathrm{~d}$ exposure and stored at $-20^{\circ} \mathrm{C}$ before analysis.

\subsection{Chemical analysis}

The plasma (50-100 $\mu \mathrm{L})$ or homogenized tissue matrix (0.05-1.32 g) was put into a $50 \mathrm{~mL}$ polypropylene centrifuge tube with $15 \mathrm{~mL}$ acetonitrile as extracting solution. The tube was stirred for 2 min on a vortex mixer, ultrasound extracted for $10 \mathrm{~min}$ and centrifuged at $8000 \mathrm{rpm}$ for $5 \mathrm{~min}$. The extraction was repeated again, the upper phase was collected and combined. The extracts were mixed with $2 \times 30 \mathrm{~mL} n$-hexane for liquid-liquid partition to remove most of lipids (only for liver and fat extraction). The upper layer was collected and filtered through $10 \mathrm{~g}$ of anhydrous sodium sulfate for dehydration and evaporated to dryness on a vacuumed rotary at $40{ }^{\circ} \mathrm{C}$. The extract was diluted with $1 \mathrm{~mL}$ methanol and passed through a $0.22 \mu \mathrm{m}$ filter membrane before measuring on HPLC/MS/MS.

Diflubenzuron, flufenoxuron and 4-chloroaniline were detected by HPLC/MS/MS. HPLC/MS/MS analyses were performed on a TSQ QUANTUM ACCESS MAX triple quadrupole MS and an Accela 600 pump/auto sampler HPLC (Thermo Electron, Hopkinson, MA). The data were collected and analyzed by the Thermo Fisher LC Quan software package (v. 2.7). A C 18 column $(2.1 \mathrm{~mm} \Phi \times 100 \mathrm{~mm} \times 5 \mu \mathrm{m}$, Thermo) was used and the flow rate in the mobile phase was $200 \mu \mathrm{L} \mathrm{min}{ }^{-1}$. For diflubenzuron and flufenoxuron analysis, the mobile phase was combined with methanol and $5 \mathrm{mmol} / \mathrm{L}$ ammonium acetate water $(90: 10, \mathrm{v} / \mathrm{v})$ while for 4 -chloroaniline analysis, methanol and $0.1 \%$ formic acid water $(80: 20, \mathrm{v} / \mathrm{v})$ were used. The analytes were detected by multiple reaction monitoring (MRM) mode using electronic spray ionization mass spectrometry (ESI-MS). The $m / z 308.99 \rightarrow 93.18$ (negative ion mode), $487.07 \rightarrow 329.13$ 
(negative ion mode), $127.93 \rightarrow 93.04$ (positive ion mode) were used for confirmation of diflubenzuron, flufenoxuron and 4-chloroaniline, respectively. The limit of detection ( $L O D, S / N=3$ ) for diflubenzuron, flufenoxuron and 4-chloroaniline were $0.005 \mathrm{mg} \mathrm{kg}^{-1}, 0.005 \mathrm{mg} \mathrm{kg}^{-1}$ and $0.001 \mathrm{mg} \mathrm{kg}^{-1}$, respectively.

\subsection{Liver histopathology}

The lizard liver was sampled at $168 \mathrm{~h}$ after dosing and stored in 4\% paraformaldehyde for histopathological analysis. Liver was processed for paraffin wax embedding. Sections were cut, stained with hematoxylin and eosin (HE), and then examined by light microscopy (Olympus DP73).

\subsection{Isolation of mRNA and quantitative real-time polymerase chain reaction}

The tissue samples were homogenized and prepared for total RNA isolation using TRIzol reagent (Life technology, Beijing, China). The cDNA was synthesized by reverse transcription reactions according to the manufacture's instructions. The reverse transcription reaction mixtures was incubated for $50 \mathrm{~min}$ at $42{ }^{\circ} \mathrm{C}$ and then heated to $95{ }^{\circ} \mathrm{C}$ for $5 \mathrm{~min}$ to inactivate the reverse transcription reaction.

The selected target genes and their respective primers are listed in Table S1. All the primers were designed by the authors using online National Center for Biotechnology Information (NCBI). Realtime PCR was performed in a MX3005P real-time quantitative polymerase chain reaction system (Stratagene, USA) in a total volume of $20 \mu \mathrm{L}$, including the SYBR Green RealMasterMix, $1 \mu \mathrm{M}$ forward primer and $1 \mu \mathrm{M}$ reverse primer. All of the samples were analyzed in triplicate and the delta-delta $\mathrm{Ct}$ method was used for results analysis. The Cyp1a and aryl hydrocarbon receptor (Ahr) mRNA expression were normalized by $\beta$-actin mRNA expression. The $\beta$ actin is selected as the most stable housekeeping gene by geNorm analysis (Table S2). A dissociation curve analysis was performed and only one peak was observed for the amplification, indicating the specific amplification of the target gene. The gene expression data showed changes relative to the control animals at $168 \mathrm{~h}$.

\subsection{Data analysis}

Tissue somatic indices $\%=$ tissue weight/body weight $\times 100$

Statistical analysis of the data was performed using one-way ANOVA, followed by an LSD Multiple Comparison Test by SPSS (version 13.0; USA). The value of $p<0.05$ was considered statistically significant.

\section{Results}

\subsection{Degradation studies of BPUs in the lizard tissues, plasma and faeces}

In this study, the degradation kinetics of the diflubenzuron is shown in Fig. 1. The concentrations of diflubenzuron in the lizards were time and tissue dependent. At the beginning of oral exposure, the uptake of diflubenzuron in the plasma and tissues were fast, and then the diflubenzuron was rapidly eliminated to a low level within $48 \mathrm{~h}$. The maximum concentrations $\left(\mathrm{C}_{\max }\right)$ of diflubenzuron in the lizard tissues and plasma were as follows: fat, brain, liver, gonad, skin, kidney, plasma, and heart. The highest levels of diflubenzuron in the fat, brain, liver, gonad, plasma and heart were shown around $24 \mathrm{~h}$ after exposure while in the skin and kidney, the $\mathrm{C}_{\max }$ was appeared within $3 \mathrm{~h}$. In the heart and kidney, the diflubenzuron was completely eliminated within $72 \mathrm{~h}$. At the end of $168 \mathrm{~h}$ exposure, the diflubenzuron concentration in the skin, plasma, liver and gonad were ranged from 0.08 to $0.59 \mathrm{mg} \mathrm{kg}^{-1}$, whereas the fat ( $\left.3.50 \mathrm{mg} \mathrm{kg}^{-1}\right)$ and brain $\left(1.83 \mathrm{mg} \mathrm{kg}^{-1}\right.$ ) could also accumulate diflubenzuron at a high level. In the faeces, the concentration of diflubenzuron was $55.60 \mathrm{mg} \mathrm{kg}^{-1}$ at $168 \mathrm{~h}$, suggesting $21.49 \%$ of administered diflubenzuron was excreted through faeces.

One of diflubenzuron metabolites, 4-chloroaniline was detected in the lizard liver, fat, skin, and gonad (Fig. S2). The concentration of 4-chloroaniline was ranged from 0.001 to $0.025 \mathrm{mg} \mathrm{kg}^{-1}$ which suggested a very low residua level. The highest concentration of 4chloroaniline was in the gonad $\left(0.025 \mathrm{mg} \mathrm{kg}^{-1}\right)$ after $12 \mathrm{~h}$ of exposure. At $168 \mathrm{~h}$, the fat tissue accumulated 4-chloroaniline at a low level and 4-chloroaniline was completely eliminated in the liver, skin and gonad. Another metabolite of diflubenzuron, 2,6difluorobenzamide was also detectable in the lizard faeces.

The concentration-time profile of flufenoxuron in the lizard tissues and plasma is shown in Fig. 2. The bioaccumulation tendency of flufenoxuron was not consistence with that of diflubenzuron. The $C_{\max }$ of flufenoxuron in the lizards was as follows: liver, heart, gonad, kidney, fat, brain, plasma and skin. There were bimodal phenomenon in the liver, brain, skin, gonad, kidney and heart. The first peak was around $24 \mathrm{~h}$ and another peak was at $120 \mathrm{~h}$ which showed the highest level of flufenoxuron. In the fat and plasma, the flufenoxuron levels increased gradually with time. The residual of flufenoxuron in the lizard kidney, fat and liver reached to values greater than $10 \mathrm{mg} \mathrm{kg}^{-1}$ at $168 \mathrm{~h}$, which indicated a high capacity of flufenoxuron to accumulate in the lizards. However, there were $32.04 \%$ of the dosed flufenoxuron was excreted through faeces, about 1.5 fold greater than that of diflubenzuron. In the faeces, one of the flufenoxuron metabolites, 2,6-difluorobenzamide was also detected (Fig. S3).

\subsection{Body weight and tissue somatic indices}

The body weight and tissue somatic indices are shown in Table 1. No significant change was observed in the body weight between the control and exposure groups. After $168 \mathrm{~h}$ diflubenzuron exposure, the liver, gonad, brain, and heart somatic indices showed a slight increase, but no significant difference was observed between the control and exposure group. The flufenoxuron treatment induced the increase of brain and kidney somatic indices while the gonad and liver coefficients were decreased. Furthermore, the hepatic index showed significantly difference compared to that in the control group.

\subsection{Liver histopathology}

In this study, a histopathological examination was carried out to determine the extent of liver toxicity as a consequence of the BPUs impacts on lizards. The morphological characteristics of the liver in the control group were normal and showed obvious cell nuclei, sinusoid, and delimited cytoplasm (Fig. 3a). In the diflubenzuron exposure group, no obvious changes have been found in the liver section compared with the control group (Fig. 3b). After the flufenoxuron treatment, the lizards showed disturbed hepatic structure with the vacuolation of cytoplasm, absence of nucleus and ambiguous sinusoid (Fig. 3c). These results suggested that the liver in the flufenoxuron exposure group showed more serious lesion.

\subsection{The gene expression of Cyp1a and Ahr mRNA in the lizard liver}

The mRNA levels of Cyp1a and Ahr genes in the lizard liver were measured after BPUs exposure (Fig. 4). Compared with the control group, no significant alteration was observed in the expression of 

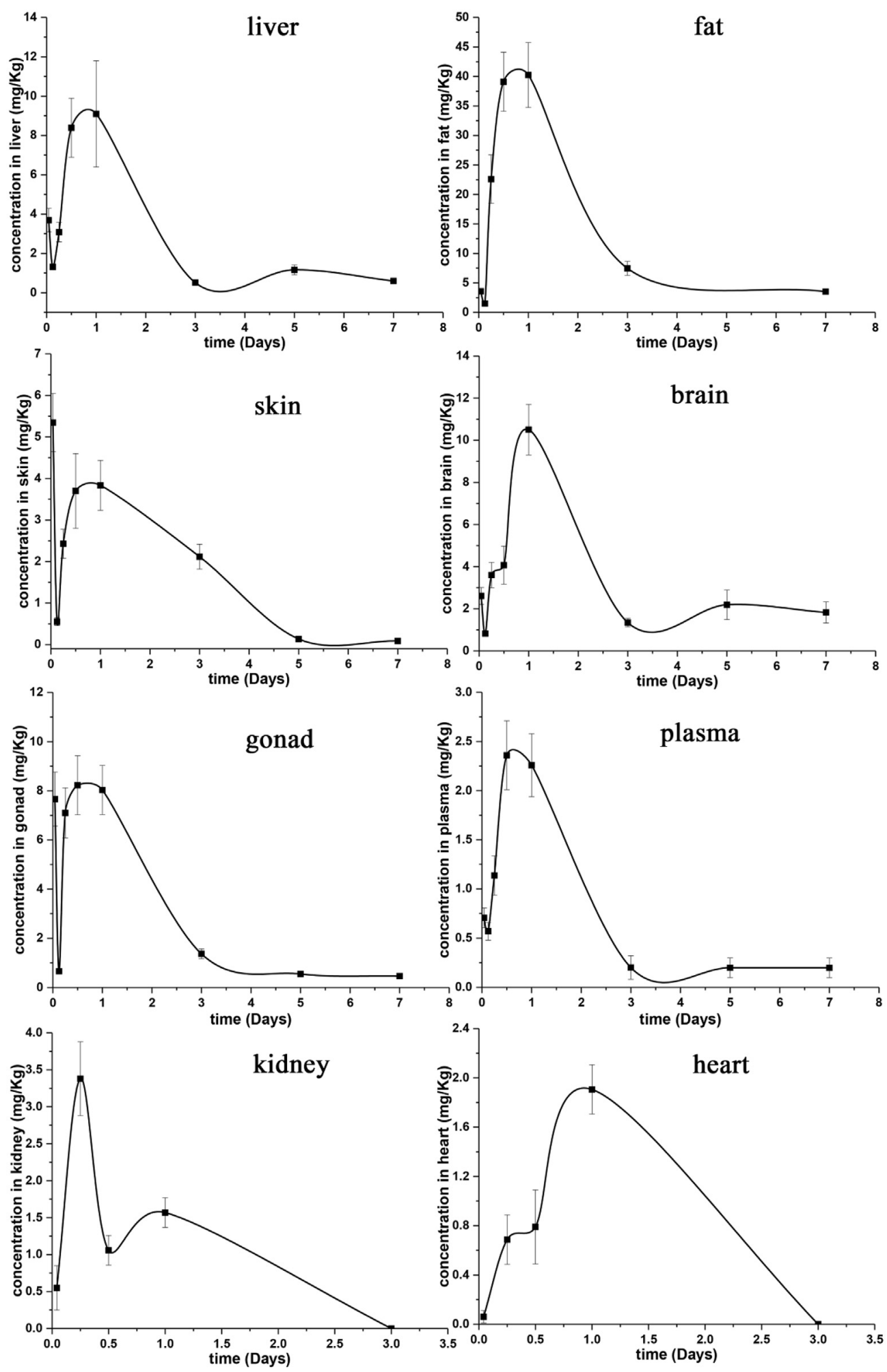

Fig. 1. The concentration-time profile of diflubenzuron in the lizard tissues and plasma after oral exposure $(n=3$, mean \pm S.E).

Cyp1a and Ahr genes after diflubenzuron exposure $(p>0.05)$. However, the mRNA levels of Cyp1a and Ahr genes were significantly up-regulated after flufenoxuron exposure. The results were consistent with the liver lesion after the BPUs treatment. The Cyp1a and $A h r$ genes were more sensitive to the flufenoxuron exposure with more serious liver lesion, compared with the diflubenzuron exposure.

\section{Discussion}

The uptake, metabolism and excretion of diflubenzuron and flufenoxuron in the lizards were different after a single dose. The 

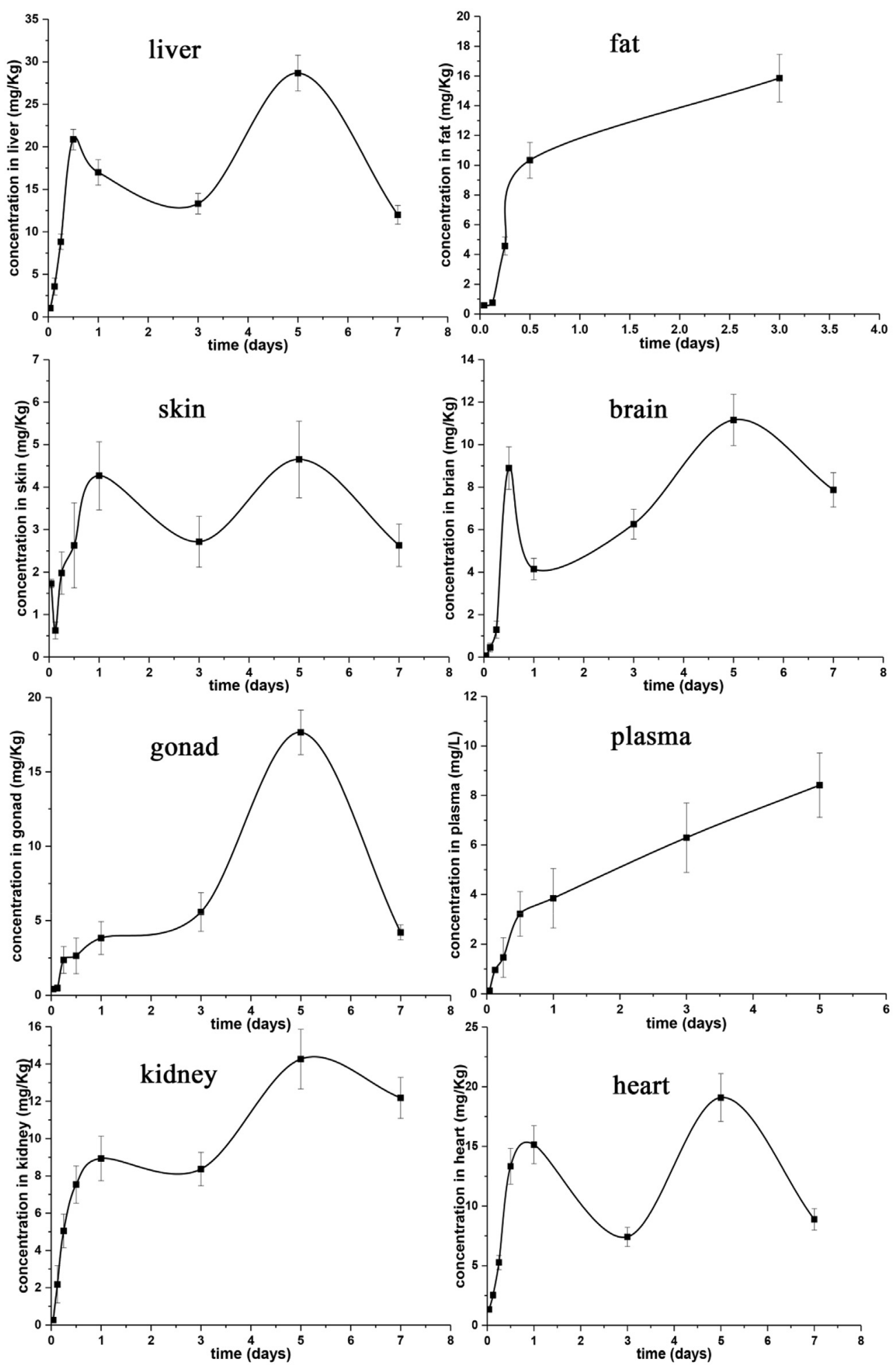

Fig. 2. The concentration-time profile of flufenoxuron in the lizard tissues and plasma after oral exposure ( $n=3$, mean \pm S.E).

diflubenzuron was eliminated rapidly in the lizard heart, kidney, gonad, skin, liver, and plasma while preferred to accumulate in the fat and brain at $168 \mathrm{~h}$. It was suggested that the diflubenzuron could across the blood-brain barrier and deposit in the brain. The higher diflubenzuron level in the brain than in the plasma was not consistent with a report by Jung et al. (2006) (Jung et al., 2006). The accumulation of diflubenzuron in the brain may demonstrate the lizard brain's limited detoxification ability and increased susceptibility. The fat and skin tissue as a depository could accumulate lipophlic compound (Kim et al., 2008). In this study, the concentration of diflubenzuron in the fat was the highest while the skin could quickly eliminate or release the diflubenzuron. Our previous study also showed that the level of lambda-cyhalothrin increased with time in the lizard fat but not in the skin (Chang et al., 2016). 


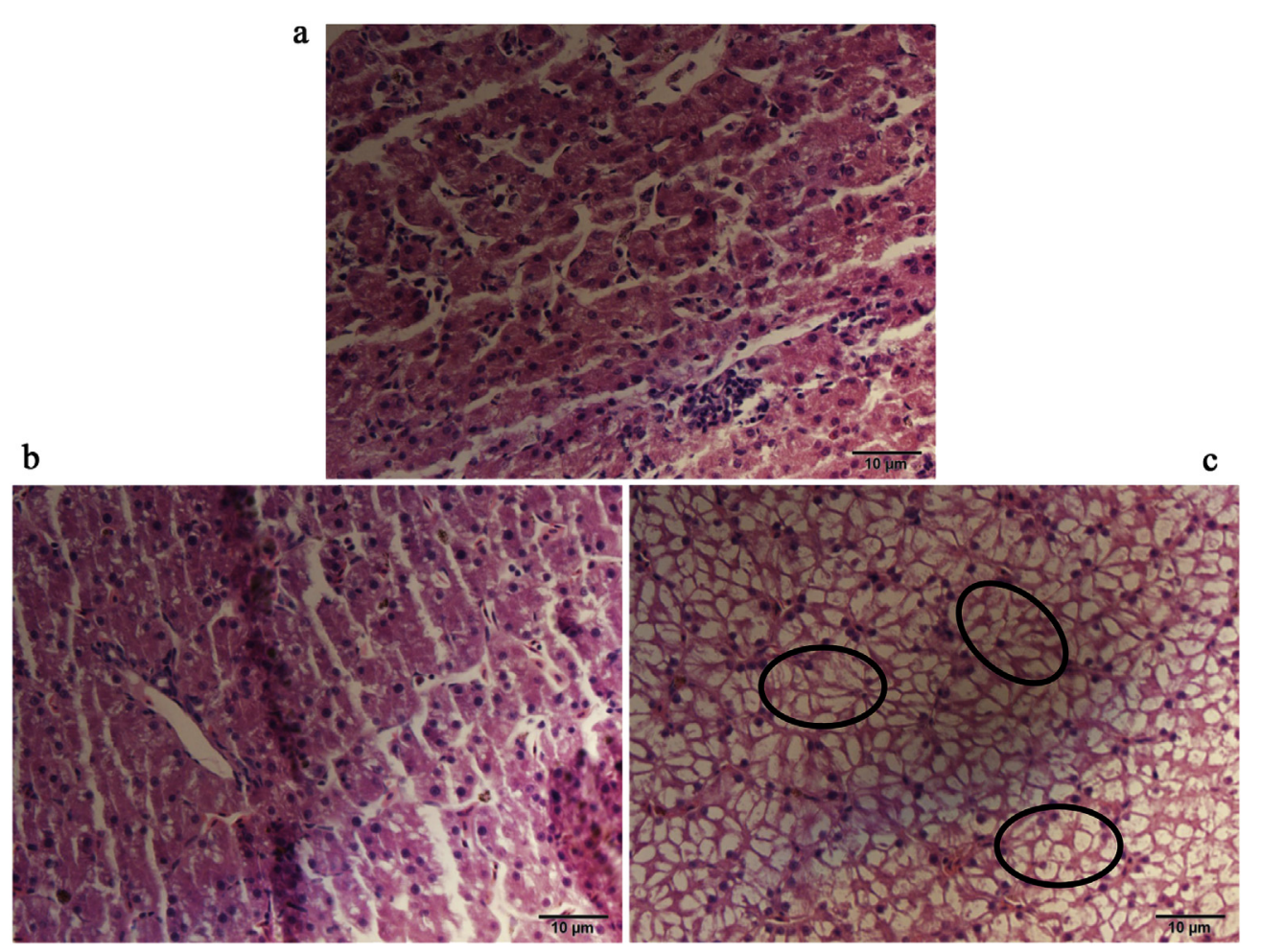

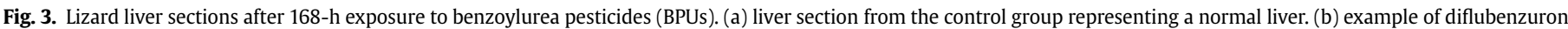
treated group. (c) example of flufenoxuron treated group showing large areas of hepatic vacuolation (black circles).

Table 1

Growth and tissue somatic indices of lizards after $168 \mathrm{~h}$ exposure to diflubenzuron or flufenoxuron.

\begin{tabular}{|c|c|c|c|c|c|c|}
\hline \multirow[t]{2}{*}{ Group } & \multirow[t]{2}{*}{ Body weight/g } & \multicolumn{5}{|c|}{ Tissue somatic indices (\%) } \\
\hline & & liver & gonad & brain & kidney & heart \\
\hline control & $3.56 \pm 0.27$ & $3.80 \pm 0.19$ & $1.04 \pm 0.12$ & $1.47 \pm 0.18$ & $0.99 \pm 0.13$ & $0.57 \pm 0.07$ \\
\hline diflubenzuron & $3.02 \pm 0.22$ & $3.73 \pm 0.25$ & $1.07 \pm 0.26$ & $1.77 \pm 0.24$ & $0.84 \pm 0.12$ & $0.70 \pm 0.10$ \\
\hline flufenoxuron & $3.64 \pm 0.25$ & $2.96 \pm 0.27^{a}$ & $0.86 \pm 0.12$ & $1.50 \pm 0.21$ & $1.21 \pm 0.16$ & $0.57 \pm 0.08$ \\
\hline
\end{tabular}

Data expressed as mean \pm S.E. of each treatment $(n=3)$.

${ }^{a}$ Statistically significant difference from the control $(p<0.05)$ (one-way ANOVA).

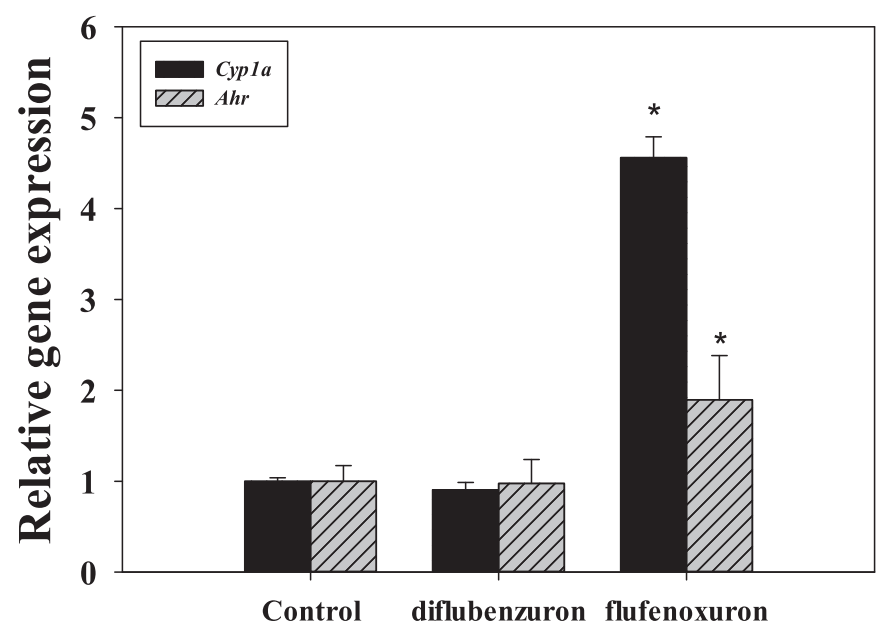

Fig. 4. The relative gene expression of Cyp1a and Ahr mRNA in the lizard liver after benzoylurea pesticides (BPUs) exposure $(\mathrm{n}=3$, mean \pm S.E). * indicates a significant difference compared to the control $(p<0.05)$ (one-way ANOVA).
Several studies have found that diflubenzuron treatment decreased the lipid level in the fat body of mealworms (Khebbeb et al., 1997) and birds (Whitmore et al., 1993). It is presumed that the high concentration of diflubenzuron in the fat tissues of lizards may be due to the decreased lipid level.

The diflubenzuron concentration in the plasma and heart is well correlated $\left(\mathrm{R}^{2}=0.85\right)$, which can be presumed that the heart transported the toxicant through blood circulation. However, a previous study has shown that a large amount of cypermethrin was retained in the lizard heart and reduced the transportation of cypermethrin in the blood (Chen et al., 2016). The different results may be explained by the different chemical-physical properties of pesticides.

The rapid elimination of diflubenzuron in the liver and kidney indicated that the metabolism enzymes in these tissues were active. Ahmed and Eid have indicated that the metabolism of diflubenzuron was mainly in the liver of fish (Ahmed and Eid, 1991). One of the possible metabolite, 4-chloroaniline was detected in the liver, although the highest concentration (1d) was less than $0.05 \%$ of the accumulated diflubenzuron. In the Atlantic salmon liver, 4chloroaniline was not a major metabolite (Schaefer et al., 1979). Diflubenzuron seems to be cleaved at the urea bridge by all animals 
(Opdycke et al., 1982a,b). It was predicted that the 4-chloroaniline may be further hydroxylated in the liver. Relatively low peak value of 2,6-difluorobenzamide in the faeces was also present to support this interpretation. As a mutagen, the low residua level of 4-chloroanilin may lead to low toxicity in the lizard tissues.

After $168 \mathrm{~h}$ exposure, only $21.49 \%$ of dosed diflubenzuron was excreted through faeces in the lizard. Combined with the low residua of diflubenzuron in tissues, it was predicted that the diflubenzuron may be excreted in the metabolites form even though 4-chloroaniline was not detected in the faeces. A previous study also showed that about $80 \%$ of the metabolites appeared to have basic diflubenzuron structure intact in the rat. In contrast, 90\% of the dosed teflubenzuron was excreted through the faeces of rat mainly in unmodified form (Koerts et al., 1997). These results indicated that the BPUs showed different metabolic routes between lizards and rats.

The tissue distribution of flufenoxuron was different compared with that of diflubenzuron. During $168 \mathrm{~h}$ degradation, bimodal phenomenon was observed in the liver, skin, brain, gonad, heart and kidney. The highest concentrations of flufenoxuron were shown at the second peak in these lizard tissues. The appearance of the second peak indicated that the elimination rate was decreased or the accumulation rate was increased around $72 \mathrm{~h}$. At the beginning of oral exposure, the gastrointestinal circulation may be responsible for the tissue accumulation of flufenoxuron (Pan et al., 2014). Due to the continuously increase of flufenoxuron in the plasma, it can be predicted that the flufenoxuron was transported to different tissues through blood circulation after $72 \mathrm{~h}$. The blood circulation was more effective route to penetrate flufenoxuron than gastrointestinal circulation.

Another important phenomenon is that the concentration of flufenoxuron was still higher than that of diflubenzuron in the lizard heart after 3 days of exposure. Human poisoning with a flufenoxuron-containing insecticide can lead to transient myocardial dysfunction in the heart (Woo and Lim, 2015). Accordingly, the relatively high residua of flufenoxuron may also induce serious effect on the lizard heart.

The metabolic capacity of flufenoxuron in the tissues may be lower than that of diflubenzuron, which induced higher residua level at the end of exposure. Because of the lower metabolic rate of flufenoxuron, it may take longer for toxicity to occur in reptiles given the same dose (Weir et al., 2016). A previous study also suggested that the enhanced toxicity of flufenoxuron to Spodoptera littoralis compared with diflubenzuron may be attributed to its lower metabolism (Clarke and Jewess, 1990). Even though the elimination rate of flufenoxuron in the lizard tissues was lower than that of diflubenzuron, the excretion of flufenoxuron in the faeces was higher. Aly and Dauterman (1992) have suggested that $47 \%$ of the administrated diflubenzuron was eliminated via the urine of the rat within $96 \mathrm{~h}$ (Aly and Dauterman, 1992). The main excretion route may be through urine rather than faeces in the lizards. Another hypothesis is that the diflubenzuron may be excreted through modified forms or metabolized into small molecular compounds, while flufenoxuron preferred to excrete in the unmodified form.

The tissue somatic indices were regarded as a predictor of the adverse effects in animals (Lopez-Islas et al., 2016). The hepatic index of lizards treated with flufenoxuron showed a significant decrease, indicating that the flufenoxuron induced more serious effect on the lizard liver. As the liver is also one of the major organs to accumulate flufenoxuron, the decrease of hepatic index can be attributed to the low degree for detoxification of flufenoxuron in the liver. Similar with the result of flufenoxuron, lizards exposed to pyrethroid pesticides showed significant decrease in the hepatosomatic index (Chang et al., 2016; Chen et al., 2016). In that case, the liver can be considered as an indicator of detoxification ability of lizards.

In consistence with the result of organ coefficient, the liver showed significant lesion in the flufenoxuron exposure group. The liver histology exhibited the decrease of cytoplasm and nuclei. The vacuolation of hepatocytes is considered to be a stage before apoptosis or a feedback effect to the lipid and carbohydrate accumulation (Abdel-Moneim et al., 2016). In contrast, the diflubenzuron exposure did not induce significant effect on the hepatocytes of lizards. There is a study suggested that diflubenzuron was devoid of any cytotoxic effects on human and rat hepatocytes (deSousa et al., 1997). It can be concluded that the flufenoxuron induced more serious impact on the hepatocytes compared with diflubenzuron.

The liver hosts a great number of enzymatically driven processes, including detoxification. The superfamily of enzymes named cytochrome P450 (CYP) is the major participant in that process (Rasmussen et al., 2016). The Ahr gene is known to influence apoptosis by regulating the expression of target genes, such as Cyp1a. (Park et al., 2005). In this study, the expression of Cyp1a and Ahr mRNA in the liver were assessed in order to proof whether the cell apoptosis was modulated by the Cyp1a and Ahr genes. No significant difference $(p>0.05)$ was observed on the expression of the Cyp1a and $A h r$ genes between the control and diflubenzuron exposure groups at $168 \mathrm{~h}$. This result may be explained by the low concentration of diflubenzuron in the lizard liver at $168 \mathrm{~h}$. Olsvik et al. have also shown that diflubenzuron was rapidly cleared from the fish and only presented small effects on the expression of genes involved in detoxification pathways (Olsvik et al., 2013). However, in the HepG2 cell, the diflubenzuron inhibited the Cyp1a expression as an Ahr antagonism after $72 \mathrm{~h}$ treatment (Ledirac et al., 2000). The influence of diflubenzuron on the Cyp1a and Ahr genes may have a connection with its concentration in non-target organisms.

The flufenoxuron treatment group showed significant upregulation of the Cyp1a and Ahr genes in the lizard liver. A previous study has shown that carbendazim with an aromatic hydrocarbon ring in the structure, is also an activator of the Cyp1a and Ahr genes (Wei et al., 2016). Combined with the decrease of hepatocytes in the liver treated with flufenoxuron, the stimulation of the $A h r$ genes has a negative effect on the cell proliferation. However, Yin et al. have suggested a positive role of Ahr in the regulation of cell proliferation and survival (Yin et al., 2016). This phenomenon indicated that the Ahr gene may play different roles on the cell apoptosis in the different organisms. In the flufenoxuron exposure group, the up-regulated Cyp1a mRNA level may be activated by the Ahr gene in the liver. As the Cyp1a gene is responsible to metabolize toxicants into less toxic metabolites (Ramakrishna et al., 2016), another speculation is that the increase of the Cyp1a gene expression may be a feedback effect to the high residua level of flufenoxuron in the liver at $168 \mathrm{~h}$. The Cyp1a and Ahr genes can be used as a biomarker to evaluate the effect of BPUs on the hepatocytes.

\section{Conclusion}

In the present study, the uptake, metabolism and toxic effect of diflubenzuron and flufenoxuron in the lizards were determined. The elimination rate between the diflubenzuron and flufenoxuron was different. The concentration of flufenoxuron in the lizard fat was 4.5 fold higher than that of diflubenzuron. In the skin, kidney and heart, the diflubenzuron reached steady states at low concentrations while the flufenoxuron treatment caused bimodal phenomenon and still showed high residua level at 168 h. 4chloroaniline is regarded as a mutagen, but it was not the major metabolite of diflubenzuron in the liver. Flufenoxuron induced more serious effect on the liver with cell apoptosis. In addition, the 
Cyp1a and Ahr genes, which are responsible to regulate the cell proliferation were significantly increased after flufenoxuron exposure. Compared with diflubenzuron, the enhanced toxicity of flufenoxuron may due to the lower metabolic rate in the lizards. The different toxic effect of the BPUs should be considered when assess the risk for reptiles and the Cyp1a and Ahr genes are important biomarkers to indicate the hepatotoxicity.

\section{Acknowledgment}

The present study was funded by the National Natural Science Foundation of China (Contract Grant number: 41301569, 21277163, 21407170 and 21477152).

\section{Appendix A. Supplementary data}

Supplementary data related to this article can be found at http:// dx.doi.org/10.1016/j.chemosphere.2017.05.009.

\section{References}

Abdel-Moneim, A.M., Essawy, A.E., Badr El-Din, N.K., El-Naggar, N.M., 2016. Biochemical and histopathological changes in liver of the Nile tilapia from Egyptian polluted lakes. Toxicol. Ind. Health 32, 457-467.

Ahmed, M.T., Eid, A.H., 1991. Accumulation of diflubenzuron bolti fish orenchromisniloticus. Nahrung Food 35, 27-31.

Aly, M.A.S., Dauterman, W.C., 1992. Bioavailability, biological-activity and charaterization of bound residues of diflubenzuron in wheat. J. Environ. Sci. Health B 27, 113-123.

Chang, J., Li, J., Wang, H., Wang, Y., Guo, B., Yin, J., Hao, W., Li, W., Li, J., Xu, P., 2016 Tissue distribution, metabolism and hepatic tissue injury in Chinese lizards (Eremias argus) after a single oral administration of lambda-cyhalothrin. Environ. Pollut. 218, 965-972.

Chen, L., Xu, P., Diao, J., Di, S., Li, R., Zhou, Z., 2016. Distribution, metabolism and toxic effects of beta-cypermethrin in lizards (Eremias argus) following oral administration. J. Hazard. Mater. 306, 87-94.

Clarke, B.S., Jewess, P.J., 1990. The uptake, excretion and metabolism of the acylurea insecticide, flufenoxuron in spodoptera-Littoralis larvae, by feeding and topical application. Pestic. Sci. 28, 357-365.

Coppen, G.D.A., Jepson, P.C., 1996. Comparative laboratory evaluation of the acute and chronic toxicology of diflubenzuron, hexaflumuron and teflubenzuron against II instar desert locust, (Schistocerca gregaria) (Orthoptera: Acrididae). Pestic. Sci. 46, 183-190.

deSousa, G., Fontaine, F., Pralavorio, M., BottaFridlund, D., Letreut, Y., Rahmani, R., 1997. Insecticide cytotoxicity and CYP1A1/2 induction in primary human and rat hepatocyte cultures. Toxicol. In Vitro 11, 451-\&.

Giari, L., Manera, M., Simoni, E., Dufuli, B.S., 2007. Cellular alterations in different organs of European sea bass Dicentrarchus labrax (L.) exposed to cadmium. Chemosphere 67, 1171-1181.

Gibbons, J.W., Scott, D.E., Ryan, T.J., Buhlmann, K.A., Tuberville, T.D., Metts, B.S., Greene, J.L., Mills, T., Leiden, Y., Poppy, S., et al., 2000. The global decline of reptiles, Deja Vu amphibians. Bioscience 50, 653-666.

Hsiao, Y.-L., Ho, W.-H., Yen, J.-H., 2013. Vertical distribution in soil column and dissipation in soil of benzoylurea insecticides diflubenzuron, flufenoxuron and novaluron and effect on the bacterial community. Chemosphere 90, 380-386.

Jung, K.H., Bong, K.K., Bartlett, M.G., Anand, S.S., Bruckner, J.V., 2006. Rapid determination of the synthetic pyrethroid insecticide, deltamethrin, in rat plasma and tissues by HPLC. J. Chromatogr. B 141-148.

Khebbeb, M.E.H., Delachambre, J., Soltani, N., 1997. Lipid metabolism during the sexual maturation of the mealworm (Tenebrio molitor): effect of ingested diflubenzuron. Pestic. Biochem. Physiol. 58, 209-217.

Kim, J.-K., Song, J.-Y., Lee, J.-H., Park, D.-S., 2010. Physical characteristics and age structure of Mongolian racerunner (Eremias argus; Larcertidae; Reptilia). J. Ecol. Field Biol. 33, 325-331.

Kim, K.-B., Anand, S.S., Kim, H.J., White, C.A., Bruckner, J.V., 2008. Toxicokinetics and tissue distribution of deltamethrin in adult Sprague-Dawley rats. Toxicol. Sci. 101, 197-205.

Koerts, J., Soffers, A., DeKraker, J.W., Cnubben, N.H.P., Rietjens, I., 1997. Metabolism of the insecticide teflubenzuron in rats. Xenobiotica 27, 801-817.

Ledirac, N., Delescluse, C., Lesca, P., Piechocki, M.P., Hines, R.N., de Sousa, G., Pralavorio, M., Rahmani, R., 2000. Diflubenzuron, a benzoyl-urea insecticide, is a potent inhibitor of TCDD-induced CYP1A1 expression in HepG2 cells. Toxicol. Appl. Pharmacol. 164, 273-279.

Lopez-Islas, M.E., Ibarra-Meza, I., Ortiz-Ordonez, E., Favari, L., Sedeno-Diaz, J.E. Lopez-Lopez, E., 2016. Liver histopatology, lipidperoxidation and somatic indices of Fulica americana in Xochimilco (urban) and Tecocomulco (rural) wetlands in the Mexico Basin. Int. J. Morphol. 34, 522-532.

Olsvik, P. A. Samuelsen, O.B., Erdal, A., Holmelid, B., Lunestad, B.T, 2013. Toxicological assessment of the anti-salmon lice drug diflubenzuron on Atlantic cod Gadus morhua. Dis. Aquat. Org. 105, 27-43.

Opdycke, J.C., Miller, R.W., Menzer, R.E., 1982a. In vivo and liver microsomal metabolism of diflubenzuron by two breeds of chickens. J. Agric. Food Chem. 30, 1227-1233.

Opdycke, J.C., Miller, R.W., Menzer, R.E., 1982b. Metabolism and fate of diflubenzuron in swine. J. Agric. Food Chem. 30, 1223-1227.

Pan, Z., Sai, F., Hong, Z.J., Hong, M., Guang, L.J., Wen, Z.G., Ning, W.Y., 2014. Application of gas chromatography-mass spectrometry in analyzing pharmacokinetics and distribution of deltamethrin in miniature pig tissues. Biomed. Environ. Sci. 426-435.

Park, K.T., Mitchell, K.A., Huang, G.M., Elferink, C.J., 2005. The aryl hydrocarbon receptor predisposes hepatocytes to Fas-mediated apoptosis. Mol. Pharmacol. 67, 612-622.

Ramakrishna, R., Bhateria, M., Singh, R., Bhatta, R.S., 2016. Evaluation of the impact of 16-dehydropregnenolone on the activity and expression of rat hepatic cytochrome P450 enzymes. J. Steroid Biochem. Mol. Biol. 163, 183-192.

Rasmussen, M.K., Theil, P.K., Oksbjerg, N., 2016. Constitutive expression of cytochrome P450 in foetal and adult porcine livers-effectss of body weight. Toxicol. Lett. 258, 87-92.

Rodriguez, E., Barrio, RJ. Goicolea, A., Peche, R, De Balugera, Z.G., Sampedro, C. 2001. Persistence of the insecticide Dimilin 45 ODC on conifer forest foliage in an Atlantic-climate ecosystem. Environ. Sci. Technol. 35, 3804-3808.

Rodriguez, E., de Balugera, Z.G., Goicolea, M.A., Barrio, R.J., 1998. HPLC/diode-array method for the determination of the pesticide diflubenzuron and its major metabolites 2,6-difluorobenzamide, 4-chlorophenylurea, and 4-chloroaniline in forestry matrices. J. Liq. Chromatogr. Rel. Technol. 21, 1857-1870.

Schaefer, C.H., Dupras, E.F., Stewart, R.J., Davidson, L.W., Colwell, A.E., 1979. Accumulation and elimination of diflubenzuron by fish. Bull. Environ. Contam. Toxicol. 21, 249-254.

Soltanimazouni, N., 1994. Effects of ingested diflubenzuron on ovarian development during the sexual-maturation of mealworms. Tissue Cell 26, 439-445.

Symmons, P., 1992. Strategies to combat the desert locust. Crop Prot. 11, 206-212.

Talent, L.G., Dumont, J.N., Bantle, J.A., Janz, D.M., Talent, S.G., 2002. Evaluation of western fence lizards (Sceloporus occidentalis) and eastern fence lizards (Sceloporus undulatus) as laboratory reptile models for toxicological investigations. Environ. Toxicol. Chem. 21, 899-905.

Wang, Y., Yu, D., Xu, P., Guo, B., Zhang, Y., Li, J., Wang, H., 2014. Stereoselective metabolism, distribution, and bioaccumulation brof triadimefon and triadimenol in lizards. Ecotoxicol. Environ. Saf. 107, 276-283.

Wei, K.L., Chen, F.Y., Lin, C.Y., Gao, G.L., Kao, W.Y., Yeh, C.H., Chen, C.R., Huang, H.C. Tsai, W.R., Jong, K.J., et al., 2016. Activation of aryl hydrocarbon receptor reduces carbendazim-induced cell death. Toxicol. Appl. Pharmacol. 306, 86-97.

Weir, S.M., Suski, J.G., Salice, C.J., 2010. Ecological risk of anthropogenic pollutants to reptiles: evaluating assumptions of sensitivity and exposure. Environ. Pollut. 158, 3596-3606.

Weir, S.M., Yu, S.Y., Knox, A., Talent, L.G., Monks, J.M., Salice, C.J., 2016. Acute toxicity and risk to lizards of rodenticides and herbicides commonly used in New Zealand. N. Z. J. Ecol. 40, 342-350.

Whitmore, R.C., Cooper, R.J., Sample, B.E., 1993. Bird fat reductions in forests treated with dimilin (R). Environ. Toxicol. Chem. 12, 2059-2064.

Woo, J.H., Lim, Y.S., 2015. Severe human poisoning with a flufenoxuron-containing insecticide: report of a case with transient myocardial dysfunction and review of the literature. Clin. Toxicol. 53, 569-572.

Yin, J.H., Sheng, B.F., Qiu, Y., Yang, K.Q., Xiao, W.D., Yang, H., 2016. Role of AhR in positive regulation of cell proliferation and survival. Cell Proliferat. 49, $554-560$. 